\title{
Agronegocios
}

Nota técnica

\section{Legislación costarricense y su relación con la bioeconomía moderna}

\author{
Costa Rican legislation and its relationship \\ with the modern bioeconomy
}

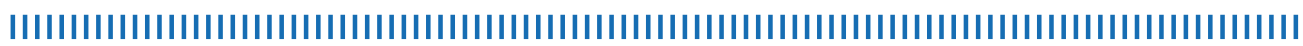

\section{José Ignacio Sánchez Gómez’}

Fecha de recepción: 27 de marzo, 2021 Fecha de aprobación: 13 de julio, 2021

Vol.7 Nº 2 Julio- diciembre 2021

Sánchez, J. (2021). Legislación costarricense y su relación con la bioeconomía moderna. Revista e-Agronegocios, 7(2). https://revistas. tec.ac.cr/index.php/eagronegocios/article/view/5637

doiDOI: https://doi.org/10.18845/ea.v7i2.5637

${ }^{1}$ Universidad de Costa Rica, San José, Costa Rica.

Correo: jose.sanchezgomez@ucr.ac.cr (iD) Orcid: https://orcid.org/0000-0003-4213-1095 


\section{Resumen}

El desarrollo histórico de la bioeconomía, se ha caracterizado por estar sumido en un proceso de constante dinamismo y evolución teórica, albergando no solo numerosas definiciones, sino también diversas estrategias que le permiten tener una aplicación apacible. Este nuevo modelo, ha llevado a los gobiernos a reconsiderar la posición que deben tomar los marcos regulatorios para alinearse al desarrollo de los tres pilares básicos de la bioeconomía (biotecnología - biomasa biodiversidad) y de esta forma, contribuir al desarrollo sostenible. El objetivo de esta investigación, es analizar las fortalezas y debilidades de la legislación costarricense en relación con el desarrollo de la bioeconomía, a través de una revisión de literatura de leyes actualizadas costarricenses del año 2017, emitidas por el Departamento de Servicios Parlamentarios de la Asamblea Legislativa. Los resultado indican un total de 58 instrumentos legales (leyes, políticas, decretos) que aportan positivamente a la producción y la conservación desde los principios bioeconómicos, una legislación limitante en la práctica de la bioeconomía y nueve herramientas que obstaculizan la producción pero aportan a la conservación, dando como conclusión, un extenso marco legal que no necesita mayor creación de leyes, sino mayormente una coordinación central y articulación de todas las herramientas con las que ya cuenta.

Palabras clave: Bioeconomía, Biotecnología, Biodiversidad, Biomasa, Legislación.

\section{Abstract}

The historical development of the concept of bioeconomy has been characterized by being immersed in a process of constant dynamism and theoretical evolution, fostering not only numerous definitions but also various strategies that allow a smoother application. This new model has led governments to reconsider the position that regulatory frameworks should take to align with the development of the three basic cornerstones of the bioeconomy (biotechnology -biomass- biodiversity) and thus contribute to achieving the sustainable development goals. The objective of this research was to analyze the strengths and weaknesses of the legislation of Costa Rica in relation to the development of the bioeconomy, through a literature review in the list of updated Costa Rican laws of the year 2017, issued by the Department of Parliamentary Services of the Legislative Assembly, finding as results, a total of 58 different legal instruments (laws, policies, decrees) that contribute positively to production and conservation from bioeconomic principles, one limiting legislation in the practice of bioeconomy, and nine legal tools that hinder production but contribute to conservation, resulting in an extensive legal framework that does not require the creation of laws, but only central coordination of all the tools it already has.

Key words: Bioeconomy, Biotechnology, Environment, Biomass, Legislation. 


\section{Introducción}

El concepto de la bioeconomía se remonta a finales de los años 1960, en el que surgió inicialmente para conferirle el valor que toman las bases biológicas de las actividades económicas (Lewandowski, 2018). No obstante, con los avances del nuevo milenio, el conocimiento biológico para la explotación comercial dejó de ser una definición suficiente al observar el potencial de la bioeconomía. Al cuantificar estas capacidades, se ha logrado estimar que más de un 90\% de los productos basados en petróleo, pueden ser remplazados por alternativas de base biológica (Borgström \& Mauerhofer, 2016), lo que ha llevado al mundo a reconsiderar nuevas posibilidades.

Una sustitución de tal magnitud, da paso a un proceso de cambio en el paradigma productivo, y esto a su vez, impulsa una etapa de generación de conocimiento y apertura hacia el entendimiento de carácter holístico y multifuncional, el cual está fundamentado en la dualidad de producción y sostenibilidad simultaneas (de Jaramillo et al., 2019), conllevando a una actualización o ajuste de los marcos legales bajo los cuales se rigen todas las actividades.

Aunque actualmente hay múltiples definiciones y una amplia internacionalización del concepto bioeconomía (Rodríguez et al., 2017), la esencia de la ideología se mantiene constante, reflejando al medio ambiente como la herramienta más importante para dinamizar las economías, aumentar la competitividad y la eco eficiencia (Staffas et al., 2013). Dichas características son producto de la fusión entre la biomasa generada por los mismos sistemas productivos y ambientales, con la adopción de biotecnologías, apoyándose en los principios de la economía circular (Aguilar et al., 2018).

Sin embargo, con la adopción de la bioeconomía como política nacional, surge también la necesidad de un marco legal acorde a las necesidades (Schütte, 2018), las cuales deben abordarse de manera tal que la transición de las actividades productivas que históricamente han sido basadas en hidrocarburos, sean apacibles hacia un nuevo modelo de revolución industrial 4.0 (Hernández \& Céspedes, 2020). El éxito bioeconómico, dependerá directamente de cómo se traduzca la definición adoptada en políticas funcionales, integrales y conectadas como un marco holístico que no obstaculice los principales pilares de la nueva propuesta (Brunori, 2013).

Esta situación implica alcanzar una simbiosis referentes a la bio seguridad, los bio riesgos, la protección de la biodiversidad, la regulación de los organismos modificados, entre muchos otros (Pyka, 2017), que vienen a ser factores determinantes para evaluar si un país está preparado para las nuevas oportunidades que trae la bioeconomía en el corto, mediano y largo plazo (Rodríguez, 2018).

Por lo tanto, considerando factores como: (a) los marcos regulatorios costarricenses 
actuales, (b) la articulación de políticas existentes, (c) la coordinación de capacidades público-privadas con las necesidades de los potenciales bio negocios, se plantea la pregunta ¿Cuáles son las fortalezas y debilidades de la legislación costarricense en relación al desarrollo de la bioeconomía?

\section{Referente teórico}

La creciente adopción de la bioeconomía a nivel mundial, ha permitido una evolución vertiginosa de su significado original hacia múltiples variaciones, dando pie a establecer y entender nuevos conceptos en una misma tasa de crecimiento como estrategias se han presentado desde los inicios del siglo XXI (Sanz-Hernández et al., 2019). Esta versatilidad que presentan los nuevos enfoques bioeconómicos, ha servido a los países para desligarse de una única definición, y ha permitido potenciar todas aquellas ventajas competitivas propias de sus sectores más fuertes (Brunori, 2013).

Este principio de aprovechamiento inteligente de los recursos, es entendido como la potenciación de las capacidades productivas (Aguilar et al., 2018), Ilevando a los países ricos en biodiversidad, a enfocar su bioeconomía a través del uso de la biomasa, mientras que los países de mayor producto interno bruto y bagaje investigativo, a concentrarse en resaltar los avances biotecnológicos (El-Chichakli et al., 2016), mientras se asegure la simbiosis y la complementariedad de estas.

Al introducir este nuevo modelo productivo en una nación y/o región, el contenido legislativo se vuelve uno de los factores más complejos de analizar, ya que la transición hacia la bioeconomía, implica una reforma al marco jurídico sobre el cual se basan todas las actividades productivas del país (Barboza Arias, 2020). Este proceso de reestructuración, debe asegurar el funcionamiento satisfactorio de las nuevas oportunidades que traen consigo los diversos tipos de bioeconomía, y fomentar el engranaje de las relaciones entre industria, academia y gobierno como una unidad complementaria (Dietz et al., 2018).

Turnheim et al., (2015) explica el concepto de la transición sostenible para fundamentar el proceso de evolución bioeconómica en términos legales, ya que este "...representa un conjunto de procesos multidimensionales y de larga duración, cuyo objetivo principal es lograr metas de sostenibilidad en un régimen o sistema determinado...". Este concepto permite comprender como la introducción de nuevas bio-tecnologías, bio-negocios, o una estrategia bioeconómica, implica realizar intervenciones regulatorias de diferente alcance, que permita acercamientos integrativos, adaptativos y proactivos de la ley (Borgström \& Mauerhofer, 2016).

El cuadro 1 muestra diferentes definiciones bioeconómicas, y permite identificar que el fortalecimiento de mercados, la inversión en I\&D, las alianzas público-privadas, com- 
petitividad, biomasa, adaptación social, bioenergía o entrenamiento vocacional (Vogelpohl \& Töller, 2021), son puntos homogéneos en los que el mundo ha concentrado sus esfuerzos bioeconómicos y debe ajustar su legislación para hacerlos efectivos dentro del entendimiento propio de bioeconomía.

\section{Cuadro 1. Definiciones nacionales de bioeconomía con enfoque holístico}

\begin{tabular}{|c|c|}
\hline País & Definición de bioeconomía \\
\hline $\begin{array}{l}\text { Costa } \\
\text { Rica }\end{array}$ & 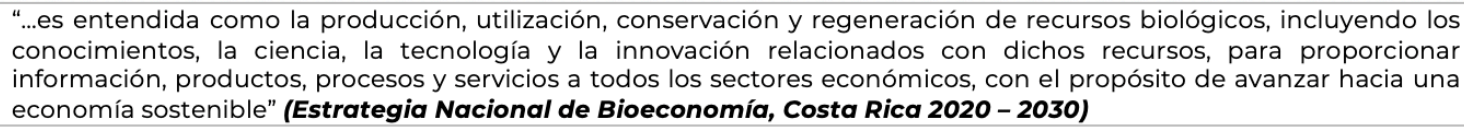 \\
\hline Austria & $\begin{array}{l}\text { "...Concepto económico que tiene como objetivo reemplazar los recursos fósiles con materias primas renovables en } \\
\text { tantas áreas y aplicaciones como sea posible, cubriendo todos los sectores económicos e industriales..." (Bioeconomy. } \\
\text { A strategy for Austria) }\end{array}$ \\
\hline Belgica & $\begin{array}{l}\text { "...Incluye la producción de recursos renovables, los residuos para ser utilizados y procesados en productos de valor } \\
\text { agregado como alimentos, concentrados animales, bioproductos y bioenergía..." (Bioeconomy in Flanders - The } \\
\text { vision and Strategy of the Government of Flanders for a sustainable and competitive bioeconomy in 2030) }\end{array}$ \\
\hline Francia & $\begin{array}{l}\text { “...abarca toda la gama de actividades vinculadas a la producción, uso y procesamiento de recursos biológicos, con el } \\
\text { propósito de brindar respuestas sostenibles a la necesidad de alimento, materias primas y energía, en armonía con los } \\
\text { ecosistemas..." (A Bioeconomic Strategy for France - Goals, Issues and Forward vision) }\end{array}$ \\
\hline Alemania & $\begin{array}{l}\text { “...La bioeconomía toma los ciclos naturales de los materiales como su punto de orientación, abarcando los sectores de } \\
\text { la economía que producen y procesan con recursos renovables..." (National Policy Strategy on Bioeconomy) }\end{array}$ \\
\hline Irlanda & $\begin{array}{l}\text { "...La agricultura y la producción de alimentos son ahora parte de una bioeconomía más grande, la cual abarca todos } \\
\text { los sectores que explotan los recursos naturales y los bio procesos, para generar productos, servicios, trabajos e } \\
\text { ingresos..." (Towards } 2030 \text { - Teagasc's Role in Transforming Ireland's Agri-Food Sector and the Wider } \\
\text { Bioeconomy) }\end{array}$ \\
\hline Italia & $\begin{array}{l}\text { “...La integración de la producción sostenible de los recursos renovables y la transformación de estos, junto con las } \\
\text { corrientes de residuos, en productos de valor agregado como alimentos, bio productos y bioenergía..." (Bioeconomy } \\
\text { in Italy: a unique opportunity to reconnect the economy, society and the environment) }\end{array}$ \\
\hline España & $\begin{array}{l}\text { "...El conjunto de las actividades económicas que obtienen productos y servicios, generando valor económico, } \\
\text { utilizando, como elementos fundamentales los recursos de origen biológico, de manera eficiente y sostenible..." (The } \\
\text { Spanish Bioeconomy Strategy - } \mathbf{2 0 3 0} \text { Horizon) }\end{array}$ \\
\hline Finlandia & $\begin{array}{l}\text { "...Una economía que se fundamente en recursos naturales renovables para producir alimentos, energía, productos y } \\
\text { servicios, todo esto bajo la independencia de los recursos fósiles, con protección ambiental y crecimiento económico } \\
\text { alineado a los principios del desarrollo sostenible..." (The Finnish Bioeconomy Strategy) }\end{array}$ \\
\hline Noruega & $\begin{array}{l}\text { “...Una producción que es sostenible, eficiente, rentable y un uso de recursos biológicos renovables para alimentación, } \\
\text { semillas, productos de salud, energía, materias primas, químicos, papel, textil y otros productos..." (Familiar Resources } \\
\text { - undream of possibilities - The Government's Bioeconomy Strategy) }\end{array}$ \\
\hline Suecia & $\begin{array}{l}\text { “...Una economía basada en una producción sostenible de biomasa que permita el crecimiento de los diferentes } \\
\text { sectores productivos y que reduzca los efectos climáticos generados por el uso de materias primas de base fósil, así } \\
\text { como una alta agregación de valor a los biomateriales que reduzca el consumo energético"(Swedish Research and } \\
\text { Innovation Strategy for a Bio-Based Economy) }\end{array}$ \\
\hline $\begin{array}{l}\text { Sur } \\
\text { Africa }\end{array}$ & $\begin{array}{l}\text { “...Las actividades que hacen uso de bio-innovaciones, basadas en recursos biológicos, materiales y procesos que } \\
\text { generan desarrollo sostenible económico, social y ambiental...(The Bio - Economy Strategy) }\end{array}$ \\
\hline $\begin{array}{l}\text { Estados } \\
\text { Unidos }\end{array}$ & $\begin{array}{l}\text { “...Una economía basada en el uso de la investigación y la innovación en las ciencias biológicas con el propósito de crear } \\
\text { actividades económicas y beneficio público..." (National Bioeconomy Blueprint) }\end{array}$ \\
\hline
\end{tabular}

Fuente: Elaboración propia con datos de BioStep, (2020) y Dietz et al., (2018), MICITT, (2020)

Aunque no existe un consenso general en la definición entendida por bioeconomía, el cuadro 1 permite observar cómo se repiten factores homogéneos en cada concepto, como es el caso de la construcción alrededor de la ideología de la transformación productiva sostenible, o su base teórica fundamentada en la triada de ambiente, economía y sociedad como partes iguales de un sistema que promueve la economía del futuro. Bajo esto, cada nación ha abordado su entendimiento por diferentes rutas pero buscando alcanzar los mismos objetivos de satisfacción de necesidades públicas y pri- 
vadas, mientras se enfrentan grandes desafíos como el crecimiento demográfico, el cambio climático, la degradación ambiental o la disminución del recurso hídrico (Maciejczak \& Hofreiter, 2015).

\section{Metodología}

Se desarrolló una investigación cualitativa basada en una revisión documental y recopilación de leyes costarricenses vinculadas directa o indirectamente a los pilares bioeconómicos de biotecnología, biodiversidad y biomasa-bioenergía propuestos por (Lewandowski, 2018). Dicha búsqueda se fundamentó en palabras clave como: conservación, protección, producción, bioeconomía, desarrollo sostenible, ambiente, agricultura, pecuaria, sostenibilidad, turismo, biotecnología, biomasa, bioenergía, y apoyo económico, en un lapso temporal indefinido que contempla legislación costarricense vigente desde el siglo XX hasta el año 2020.

Específicamente, el análisis se basa en la revisión del listado de leyes actualizadas costarricenses emitida por el Departamento de Servicios Parlamentarios en el año 2017, perteneciente a la Unidad de Actualización Normativa de la Asamblea Legislativa Costarricense. Para este análisis, se relacionan los listados de dicho documento con las tres categorías consideradas como pilares de la bioeconomía. Sumado a esto, se agrega a la discusión tres ejes transversales. Estos son: 1) leyes vinculantes a la bioeconomía específicamente para el sector turismo, debido al elevado importe económico que significa este campo para el país y la extensa relación con la biodiversidad y el desarrollo rural. 2) Manejo pecuario, por su estrecha relación entre la actividad ganadera y la biomasa-bioenergía, y finalmente, 3) incentivos económicos sujetos al desarrollo de posibles nuevos bio emprendimientos.

El proceso de análisis para esta investigación se centró en preseleccionar todas aquellas leyes costarricenses que actualmente estén dentro del período de vigencia, de publicación y/o de actualización (independientemente de su año de creación) y que dentro de su título se haga referencia a las palabras claves mencionadas anteriormente. Como segundo proceso de escrutinio, se detallaron a mayor profundidad las leyes seleccionadas en el Sistema Costarricense de Información Jurídica (disponible en línea) para corroborar que dentro del texto de la ley, la ficha de la norma o sus artículos, efectivamente se referencie directa o indirectamente los temas relacionados a bioeconomía, conservación y/o producción sostenible (pilares bioeconómicos). Una vez contemplada toda la legislación, se clasificó en tres cuadros, correspondientes a cada pilar bioeconómico y se procedió a categorizar de acuerdo al contenido de su texto, si las leyes aportan u obstaculizan al marco del desarrollo de la bioeconomía en Costa Rica. 


\section{Resultados}

\section{Pilares bioeconómicos}

El papel de la biotecnología dentro de los alcances de la bioeconomía, implica no solo el crecimiento industrial sino que pone a disposición múltiples opciones innovadoras para cumplir los objetivos del desarrollo sostenible (Lokko et al., 2018). Esta característica, la ha posicionado como una de las bases fundamentales para asegurar el éxito de los nuevos modelos bioeconómicos, ya que gracias a esta se ha logrado verdaderamente optimizar el uso de los recursos naturales dentro de los procesos productivos (Wield, 2013), impactando áreas como la producción de alimentos, la industrias farmacéuticas o la preparación de materias primas biológicas (Kircher et al., 2020).

Este sinfín de aplicaciones involucra una afectación directa e indirecta sobre los diferentes actores de las cadenas de valor, por tanto, surge una necesidad imperante de regulación legal dentro de los procesos de obtención de nuevos bio productos y toda la cadena hasta los consumidores. Para evaluar esta condición en la legislación costarricense, se muestra el cuadro 2, el cual detalla la posición que toman las diferentes herramientas legales con respecto al tema biotecnológico, y su posición de apoyo o limitación a la bioeconomía costarricense.

Cuadro 2. Legislación costarricense de vinculo biotecnológico - bioeconómico

\begin{tabular}{|c|c|c|}
\hline Leyes de apoyo a la bioeconomía (10) & Producción & Conservación \\
\hline $\begin{array}{l}\text { - Ley de Promoción del Desarrollo Científico y Tecnológico (7169) · Ley de Protección Fitosanitaria } \\
\text { (7664) - Ley de Aprobación del Protocolo de Cartagena sobre seguridad de la Biotecnología (8537) } \\
\text { - Aprobación de la adhesión de Costa Rica al Tratado de Budapest sobre el reconocimiento } \\
\text { internacional del depósito de microorganismos a los fines del procedimiento en materia de } \\
\text { patentes (8633) . Aprobación del Tratado Internacional sobre los recursos fitogenéticos para la } \\
\text { alimentación y la agricultura (8539) - Ley del Instituto Nacional de Innovación y Transferencia en } \\
\text { Tecnología Agropecuaria (8149). Aprobación de los Estatutos y el Protocolo del Centro } \\
\text { Internacional de Ingeniería Genética y Biotecnología (7613). Aprobación del Acuerdo entre el } \\
\text { Gobierno de la República de Costa Rica y el Instituto Internacional de Recursos Genéticos de } \\
\text { Plantas (IPGRI) para el establecimiento y operación de una oficina IPGRI en Costa Rica (854) · Ley } \\
\text { de Procedimientos de Observancia de la Propiedad Intelectual (8039) - Ley Reguladora de } \\
\text { Investigación Biomédica (9234) }\end{array}$ & Aporta & Aporta \\
\hline \multicolumn{3}{|l|}{ Políticas de apoyo a la bioeconomía (4) } \\
\hline $\begin{array}{l}\text { - Decreto Ejecutivo 40050-MINAE-MAG "Reglamento de Biocombustibles Líquidos y sus Mezclas" } \\
\text { (2016) · Programa Nacional de Biocombustibles (2018) · Política Nacional de Sociedad y Economía } \\
\text { basada en el Conocimiento 2017-2030 - Plan Nacional de Ciencia, Tecnología e Innovación 2015- } \\
2021 \text { (2015) }\end{array}$ & Aporta & Aporta \\
\hline \multicolumn{3}{|l|}{ Limitantes a la bioeconómica (1) } \\
\hline - Trámites de las solicitudes de registro de agroquímicos (8702) & Limita & Limita \\
\hline
\end{tabular}

Fuente: elaboración propia con datos de SCIJ (2020), Asamblea Legislativa República de Costa Rica (2020), UNED (2012), ICT (2009) y Senara (s. f.).

En cuanto a la biomasa - bioenergía, Scarlat et al., (2015) y Lago et al., (2019) la presentan como otro de los pilares básicos bioeconómicos, ya que tiene un rol de proveedora 
de materias primas biológicas, las cuales van estrechamente ligada con la circularidad económica, sustituyendo la filosofía productiva de "tomar, fabricar y eliminar" e introduciendo nuevos conceptos como el "uso en cascada" (Haberl \& Geissler, 2000), que sienta sus bases en la utilización eficiente del material considerado como "residuo" para extender la disponibilidad de biomasa dentro del sistema por mayor tiempo, mientras disminuye el impacto en el ambiente (Lago et al., 2019).

Considerando estos antecedentes y el potencial de surgimiento de nuevos campos de conocimiento como los biomateriales o la química verde (Scarlat et al., 2015), es que también se evalúa la complementariedad legal que tiene el país para fomentar la innovación biológica y asegurar la disponibilidad de biomasa sostenible. Esto se presenta en el cuadro 3, el cual detalla una serie de leyes y documentos legales que aportan herramientas para apoyar la producción y la conservación desde el enfoque bioeconómico.

\section{Cuadro 3. Legislación costarricense de vinculo biomasa - bioeconomía}

\begin{tabular}{|c|c|c|}
\hline Leyes de apoyo a la bioeconomía (7) & Producción & Conservación \\
\hline $\begin{array}{l}\text { - Ley de Manejo Integral de Desechos (8839) - Ley de Promoción del Transporte } \\
\text { Eléctrico (4905) - Ley de Generación Eléctrica autónoma o Paralela (7200) } \\
\text { Aprobación del Segundo Protocolo al Tratado Marco del Mercado Eléctrico de } \\
\text { América Central } \mathbf{( 9 0 0 4 )} \cdot \text { Ley para regular el mejor aprovechamiento de la luz solar } \\
\mathbf{( 7 4 4 6 )} \text { - Regulación del uso racional de la energía (7447) - Ley de creación del ICE } \\
(\mathbf{4 4 9 )}\end{array}$ & Aporta & Aporta \\
\hline \multicolumn{3}{|l|}{ Políticas de apoyo a la bioeconomía (6) } \\
\hline $\begin{array}{l}\text { - Plan Nacional de Energía 2015-2030 (2015) · Plan Nacional de Descarbonización · } \\
\text { Estrategia Nacional de sustitución de plásticos de un solo uso 2017-2021 (2017) · } \\
\text { Política Nacional de Producción y Consumo Sostenible 2018-2030 (2018) · Plan de } \\
\text { Promoción y Desarrollo de Fuentes Renovables no Convencionales · Plan Nacional } \\
\text { de Gestión de Residuos } 2016-2021 \text { (2016) }\end{array}$ & Aporta & Aporta \\
\hline
\end{tabular}

Fuente: elaboración propia con datos de SCIJ,(2020), Asamblea Legislativa República de Costa Rica (2020), UNED (2012), ICT (2009) y Senara (s. f.)

Finalmente, el papel más importante en los cimientos de la bioeconomía lo toma la biodiversidad (Scarlat et al., 2015) ya que esta complementa de manera perfecta los avances biotecnológicos, y brinda a la biomasa los recursos para la creación de materias primas y esto a su vez para la transformación de energías (Valli et al., 2018). Desde el ámbito legal, este pilar implica dos caras de una misma moneda, ya que la ley debe asegurar su protección y preservación como base fundamental de la sostenibilidad, pero también debe promover su compromiso con el uso sin impedir que los avances generados por la innovación sean obsoletos. Esta situación se especifica en el cuadro 4 , donde se muestra el marco legal costarricense concerniente a la relación bioeconomía - biodiversidad. 


\section{Cuadro 4. Legislación costarricense de vinculo biodiversidad - bioeconomía}

\begin{tabular}{|c|c|c|}
\hline Leyes de apoyo a la bioeconomía (14) & Producción & Conservación \\
\hline 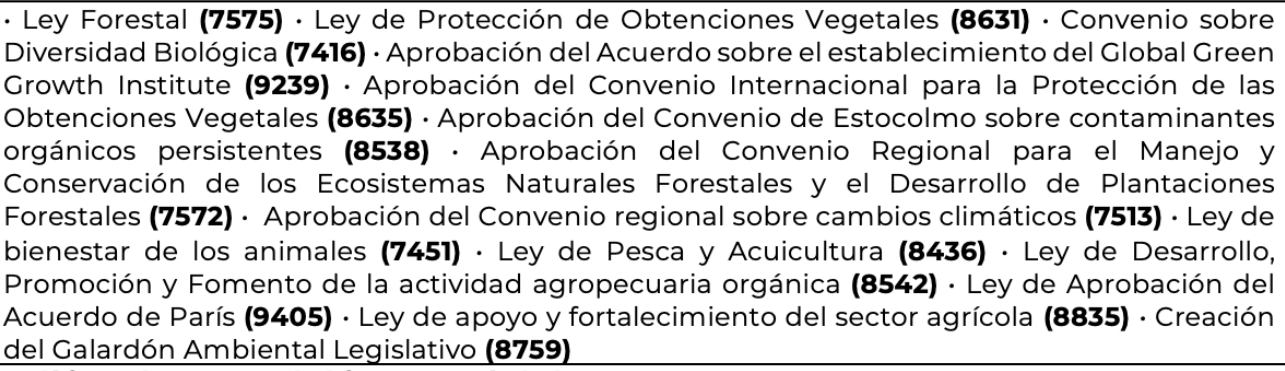 & Aporta & Aporta \\
\hline \multicolumn{3}{|l|}{ Políticas de apoyo a la bioeconomía (17) } \\
\hline 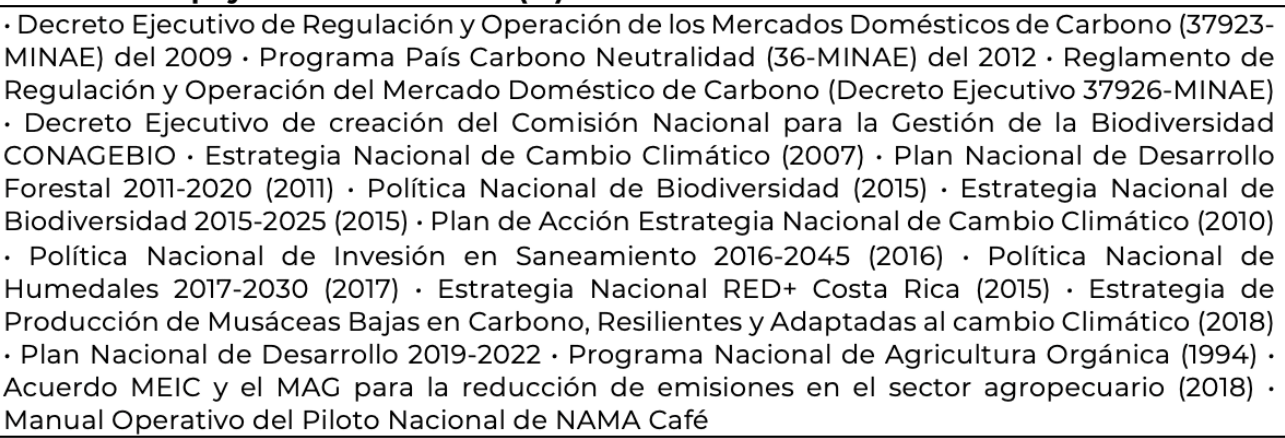 & Aporta & Aporta \\
\hline \multicolumn{3}{|l|}{ limitantes a la bioeconómica (9) } \\
\hline $\begin{array}{l}\text { - Ley de Conservación de la Fauna Silvestre (6919) · Servicio Nacional de Parques Nacionales } \\
\text { (6184) · Ley Orgánica del Ambiente (7554) · Ley de Conservación de la Vida Silvestre (7317) } \\
\text { Aprobación del Convenio Constitutivo de la Comisión Interparlamentaria Centroamericana de } \\
\text { Ambiente y Desarrollo (8210) · Ley de Uso, Manejo y Conservación de Suelos (7779) · Convenio } \\
\text { para la Conservación de la Biodiversidad y Protección de Áreas Silvestres prioritarias en } \\
\text { América Central (7433) · Ley de Biodiversidad (7788) · Decreto Ejecutivo de Regulación de la } \\
\text { Biodiversidad (2008) }\end{array}$ & Limita & Aporta \\
\hline
\end{tabular}

Fuente: elaboración propia con datos de SCIJ,(2020), Asamblea Legislativa República de Costa Rica (2020), UNED (2012), ICT (2009) y Senara (s. f.)

\section{Discusión}

\section{Desarrollo biotecnológico}

Los resultados presentados en el cuadro 2 permiten observar como Costa Rica ha llevado a cabo esfuerzos por regular la actividad y colocar inversiones estratégicas en actividades científicas y de innovación, con el fin de apoyar el auge biotecnológico y el desarrollo científico. Un ejemplo de esto se da particularmente en el año 2018, cuando el país destinó una suma cercana a los 1836 millones de dólares (3,06\% del PIB) para actividades científicas y tecnológicas (MICITT, 2019) colocándose como un vanguardista en la región, solo superado por Chile y México en los índices de innovación (OMPI, 2020) 
Al contrastar el aporte económico de Costa Rica específicamente a la I\&D (0,39\% del PIB) contra países de mayor historial tecnológico como Corea $(4,30 \%)$ o Japón $(3,40 \%)$ se evidencia que aún el país está en etapas tempranas de desarrollo (UNESCO, 2020), no obstante, cuenta con al menos diez leyes directas de vinculo biotecnológico - bioeconómico y cuatro políticas diferentes que le aportan herramientas legales a la producción y conservación de los recursos a través del uso inteligente de los mismos. Se debe realizar una mención especial a la Ley 8039 sobre propiedad intelectual, y la Ley 9234 de investigación biomédica, las cuales, apoyan cada una desde su área al factor productivo, pero no tienen una relación obstaculizadora o directa con la conservación, por lo que, para este artículo se consideran aportes legales positivos a la bioeconomía.

El caso de la Ley 8702 de registro de agroquímicos, sobresale como una situación anómala dentro del marco regulatorio analizado, y se presenta como una limitante a la producción y conservación por su sistema actual de ejecución, mas no por su contenido. Esta ley desde el ámbito teórico expresa en su artículo 1 "...tramitar las solicitudes de registros de nuevos plaguicidas o de modificaciones a registros vigentes..." (Sistema Costarricense de Información Jurídica, 2009) sin embargo, es víctima de una fuerte limitante burocrática que le impide la autorización o el ingreso de nuevos productos a suelo costarricense, generando como consecuencia para el ambiente, un daño mayor al obligar a los agricultores a depender de mayores volúmenes de productos no óptimos que afectan directamente a la competitividad internacional (Chacón, 2018).

En cuanto a los programas, decretos o políticas, la revisión de información permitió establecer parámetros positivos para la bioeconomía, ya que a través de estas, se da soporte a áreas como el biorrefinamiento de combustibles, que es vital en la descarbonización de la atmosfera, la economía basada en el conocimiento o el desarrollo de la innovación y la tecnología (RECOPE, 2018), ambos puntos de convergencia en todas las estrategias mundiales desarrolladas a través de objetivos estratégicos.

\section{Biomasa - Bioenergía}

La recopilación de leyes mostradas en el cuadro 3 sobre manejo y aprovechamiento de la Biomasa-Bioenergía dentro del aparato económico del país, permite concluir que este aún se encuentra en etapas iniciales, no obstante, Rivera-Tenorio \& Moya, (2020) y Chavarría et al., (2020) presentan a través de casos puntuales de éxito, evidencias del extenso potencial costarricense para aplicar fuentes renovables de energía y adaptación de residuos como nueva biomasa dentro de los sistemas productivos agrícolas e industriales.

Las trece diferentes iniciativas legales que rodean este pilar bioeconómico, tienen principalmente un impacto positivo en la adopción del modelo bioeconómico desde el ámbito teórico, ya que promulgan la protección de los recursos naturales, la circula- 
rización de la economía, la descarbonización del ambiente o el uso de los biocombustibles, todo esto sin obstaculizar el desarrollo productivo sostenible, aunque se debe hacer la salvedad en casos específicos, su aplicación en el corto y mediano plazo puede ser un reto para el sector productivo debido a las implicaciones técnicas que conllevaría para cada actividad económica.

Dichas dificultades son más notorias en casos como la Ley 4905 de Promoción del transporte eléctrico, la cual se centra en incentivar su uso dentro de la población; o en la Estrategia Nacional sobre la sustitución de plásticos de un solo uso, las cuales, deben ser abordadas de manera paulatina, ya que los fuertes ajustes estructurales y económicos que implica el proceso de sustitución pueden traer impactos contraproducentes en los diferentes sectores. Imbert et al., (2017) explica esta condición a través de la necesidad de la progresión controlada, donde en un análisis de la estrategia bioeconómica Alemana e Italiana, encuentra dentro de sus diferencias, en el acercamiento teutón, un proceso controlado por una coordinación horizontal desde los niveles ministeriales más elevados, hasta la población, haciendo más pacífica la adopción de nuevos bio-productos.

\section{Conservación de biodiversidad y ecosistemas}

Desde la coyuntura del ámbito legal y ambiental, el cuadro 4 permite reafirmar los argumentos de Steinberg et al., (2001) sobre la postura vanguardista y defensora que adopta Costa Rica para perseguir el objetivo de la preservación de la biodiversidad y de los múltiples ecosistemas que alberga. Dicha posición, ha tomado un rol importante a nivel internacional, al convertirse en un marco de referencia para muchas otras naciones que abogan por salvaguardar el ambiente y potenciar el desarrollo sostenible (Blum, 2008).

Este cuadro, expone una amplia gama de leyes con una orientación estrictamente proteccionista, como es caso de las Leyes 7788 de Biodiversidad, 6919 de Conservación de la Fauna Silvestre, 7554 Ley Orgánica del Ambiente, o la Ley 7317 de Conservación de Vida Silvestre; las cuales, aunque en primera instancia obstaculizan el eje productivo de la dualidad "producción - conservación", en un esquema mayor, no toma realmente una posición de limitante bioeconómica, sino que todas estas leyes, únicamente se encargan de delimitar los alcances físicos de los sectores productivos y no son un impedimento al uso de los recursos en forma sostenible dentro de la totalidad de la extensión del país, sino únicamente en zonas previamente definidas como protegidas, reafirmando que la conservación también es un principio básico de la bioeconomía.

Finalmente, este apartado se completa con un número importante de decretos, programas, planes y estrategias que dan fundamento teórico y práctico al modelo bioeconómico costarricense, mostrando que temas como la regulación de mercados de 
carbono o el saneamiento de aguas residuales, indirectamente también son aspectos claves para una aplicación holística y productiva.

\section{Sector turismo}

La importancia que tiene el desarrollo del turismo rural dentro del contexto de la bioeconomía, surge de la simbiosis entre la explotación sostenible de los atractivos naturales, con los componentes culturales, históricos y sociales del área donde se desarrollan (Hadad, 2019). Este tipo de turismo, permite brindarle oportunidades de crecimiento y mejor calidad de vida a las pequeñas poblaciones alejadas del área industrializada, mientras que a su vez dinamiza las económicas locales y de las regiones aledañas (Hadad, 2019). Simultáneamente, al potenciar el turismo como parte del marco constituyente de la bioeconomía, el país ataca directamente problema sociales como la migración a zonas urbanas, el desempleo o la baja industrialización (Martín, 2013).

Estos motivos, justifican introducir la relación entre bioeconomía y el turismo costarricense dentro de este análisis, especialmente cuando la magnitud de importe económico $(6,3 \%$ del PIB) y el volumen de mano de obra directa e indirecta $(20,8 \%$ del total de M.O del país) que alberga son tan elevadas (Benavides Vindas, 2020) \& (ICT, 2020). Afortunadamente, la legislación que vincula ambos campos ha sido construida históricamente en función de la preservación de los recursos, y se ha publicitado mundialmente como una zona de refugio para la flora y la fauna, por lo que dicho marco regulatorio se apega a los límites de un modelo bioeconómico.

La principal legislación con la que cuenta Costa Rica para fomentar este tema nace desde la Ley Orgánica del Instituto Costarricense de Turismo (8694) y se apoya en otras leyes como: la Ley de Fortalecimiento de la Industria Turística (8694), Programa de apoyo y reactivación de las MIPYMES del sector turismo (9339), Contrato de préstamo NN 1824/OC-CR y su anexo entre el Gobierno de la República de Costa Rica y el Banco Interamericano de Desarrollo, para financiar el programa de turismo en áreas silvestres (8967), Incentivo de la responsabilidad social corporativa turística (8811), Fomento de Turismo Rural Comunitario (8724), Fortalecimiento del desarrollo de la industria turística nacional (8684), Aprobación del Convenio para el establecimiento de la zona de turismo sustentable del Caribe y su protocolo (8879) o el Plan Nacional de Turismo Sostenible 2010 - 2016.

\section{Manejo Pecuario}

La temática referente al marco regulatorio para el manejo pecuario y su vínculo con la bioeconomía, radican en gran parte por su relación con la biomasa residual generada por la actividad ganadera y la conservación del ambiente mediante el desarrollo rural. Todos los marcos regulatorios bajo los que se rigen estas actividades productivas en 
el país, han contribuido de manera positiva en la protección de los animales utilizados para trabajo, y han sentado bases técnicas para el apoyo bioeconómico mediante diversas estrategias como la Nama Ganadería o la Ganadería Baja en Carbono, dando como resultado para el criterio de análisis de este artículo, que dicha legislación aporta a la bioeconomía desde el ámbito productivo y de conservación.

Los principales instrumentos legales que se encuentran en este apartado son: las acciones nacionales de mitigación (Nama Ganadería), Estrategia Nacional de Ganadería Baja en Carbono, Ley de bienestar animal (7451), Ley de desarrollo, promoción y fomento de la actividad agropecuaria orgánica (8542), Control de ganado bovino, prevención y sanción de su robo, hurto y receptación (8799), Desarrollo, promoción y fomento de la actividad orgánica (8591), Ley General de Servicio Nacional de Salud Animal (8495), Creación de la Corporación Ganadera (7837), Creación del programa de reconversión productiva del sector agropecuario (7742), Ley de fomento a la producción agropecuaria (7064), Control, elaboración y expendio de alimentos para animales (6883), Ley de fomento a la actividad porcina (6433), Ley sobre normas referentes al abastecimiento de ganado vacuno para el consumo nacional y la exportación (6247), Medidas sanitarias promovidas en relación con mejoramiento de producción animal y su repercusión directa en la salud del hombre (6243), Ley reguladora para la exportación de ganado vacuno hembra (5098), Declaratoria contra la economía nacional el dar muerte a las crías recién nacidas del ganado de leche (4443) o la Ley de Exportación de ganado vacuno (4412).

\section{Incentivos económicos}

Para concluir este apartado, se plantea un último conglomerado de leyes costarricenses con un importante vínculo para el desarrollo de la bioeconomía. Esta legislación es claves para el futuro de los bio emprendimientos, ya que tiene la capacidad de aportar factores de financiamiento y acompañamiento técnico en múltiples campos simultáneamente.

Dentro de las principales leyes que se destacan para esta labor, se encuentran: la Ley 8932 sobre Exoneración del pago de tributos de sistemas de tratamiento de aguas residuales para contribuir a mitigar la contaminación del recurso hídrico y mejorar la calidad del agua, la Ley 8634 del Sistema de Banca para el Desarrollo, la Ley 8663 sobre Fortalecimiento del Programa Integral de Mercadeo Agropecuario, la Ley 8262 de Fortalecimiento de las Pequeñas y Medianas Empresas, o la Ley de Fomento a la Industria Rural (6847). 


\section{Conclusiones}

La construcción nomotética de las últimas décadas, permite amalgamar positivamente un vasto número de instrumentos legales con los principios básicos que envuelven a la bioeconomía moderna. Dichos estatutos, aunque originalmente no fueron concebidos bajo el marco teórico de la bioeconomía, si se adecúan directamente a los principios del desarrollo sostenible y uso inteligente de recursos, lo cual fundamenta la bioeconomía basada en conocimientos (Urmetzer \& Pyka, 2017).

Las principales fortalezas que se encontraron en esta investigación son: 1) la elevada cantidad de marcos regulatorios en cada flanco; específicamente 58 instrumentos legales brindando contexto a los tres pilares principales, y al menos 30 legislaciones que se identifican con el modelo desde los ejes transversales de turismo, manejo animal e incentivos económicos. 2) Aunque las normativas muestran un enfoque proteccionista del ambiente, no se limita ni interrumpe en gran medida a la producción, sino que únicamente la ubica dentro de límites y alcances para asegurar la sostenibilidad de recursos. 3) A pesar de que la bioeconomía en Costa Rica es reciente, y está en pleno auge mundial, esta no ha postergado su implementación, sino que ha intentado aumentar sus fronteras y concentrar esfuerzos en ligar el concepto con la competitividad y la innovación.

Respecto a las debilidades identificadas, se presentan: 1) la aplicabilidad real de las leyes en el corto y mediano plazo, las cuales desde su fundamento teórico son positivas, pero ante la ausencia de un plan de transición paulatina, el resultado puede ser un efecto contraproducente al desarrollo económico, y 2) El reto técnico y administrativo que implica para el estado la articulación de los marcos regulatorio bajo un único esquema bioeconómico de enfoque holístico, ya que todo el proceso necesita una evaluación constante para garantizar su correcto funcionamiento en el paso del tiempo.

Finalmente, este diagnóstico lleva a plantear las condiciones sobre el futuro de la bioeconomía en Costa Rica, la cual necesita mantener un crecimiento constante que garantice una sustitución de bienes y servicios hacia bioproductos y bioservicios. Es necesario aumentar el respaldo económico de bioemprendimientos y el acceso a biotecnología, pero también debe iniciar el proceso de concientización poblacional que muestre las implicaciones de no adoptar los recursos naturales como la base primaria para todas las actividades, ya que la sostenibilidad debe ser vista como una nueva forma de vida (El Ghorab \& Shalaby, 2016), más allá de una acción política específica. 


\section{Literatura citada}

Aguilar, A., Wohlgemuth, R., \& Twardowski, T. (2018). Perspectives on bioeconomy. New Biotechnology, 40, 181-184. https://doi.org/10.1016/j.nbt.2017.06.012

Asamblea Legislativa República de Costa Rica. (2017). Listado de Leyes Actualizadas. http://www.asamblea.go.cr/sd/SiteAssets/Lists/Consultas\%20 Biblioteca/EditForm/LISTADO\%20\%20DE\%20\%2OLEYES\%20\%20 ACTUALIZADAS.pdf

Barboza Arias, L. M. (2020). Nota técnica sobre la formulación de la Estrategia Nacional de Bioeconomía en Costa Rica |. e-Agronegocios, 7(1), 21-37. https:// doi.org/ 10.18845/ea.v7i1.5190

Benavides Vindas, S. (2020). Vista de El aporte del turismo a la economía costarricense: Más de una década después. Economia y Sociedad, 25(57). https://doi.org/10.15359/eys.25-57.1

BioStep. (2020). Bioeconomy strategies. http://www.bio-step.eu/background/ bioeconomy-strategies.html

Blum, N. (2008). Environmental education in Costa Rica: Building a framework for sustainable development? International Journal of Educational Development, 28(3), 348-358. https://doi.org/10.1016/j.ijedudev.2007.05.008

Borgström, D. S., \& Mauerhofer, D. V. (2016). Developing law for the bioeconomy. Journal of Energy \& Natural Resources Law, 34(4), 373-406. https://doi.org/10.1 080/02646811.2016.1200349

Brunori, G. (2013). Biomass, Biovalue and Sustainability: Some Thoughts on the Definition of the Bioeconomy. EuroChoices, 12(1), 48-52. https://doi. org/10.1111/1746-692X.12020

Chacón, V. (2018). MAG busca control total del registro de agroquímicos. Semanario Universidad. https://semanariouniversidad.com/pais/mag-buscacontrol-total-del-registro-de-agroquimicos/

Chavarría, H., Trigo, E., \& Martínez, J. F. (2020). Políticas y Negocios para la Bioeconomía en ALC: Un proceso en marcha: C3-BIOECONOMY: Circular and Sustainable Bioeconomy, 1, 72-88 / 69-85 (EN). https://doi.org/10.21071/c3b. vi1.13150 
de Jaramillo, E. H., Henry, G., \& Trigo, E. (Eds.). (2019). La bioeconomía. Nuevo marco para el crecimiento sostenible en América Latina: Bioeconomy. New framework for sustainable growth in Latin America (1.a ed.). Pontificia Universidad Javeriana. https://doi.org/10.2307/j.ctvkwnpxt

Dietz, T., Börner, J., Förster, J. J., \& Von Braun, J. (2018). Governance of the Bioeconomy: A Global Comparative Study of National Bioeconomy Strategies. Sustainability, 10(9), 3190. https://doi.org/10.3390/su10093190

El Ghorab, H. K., \& Shalaby, H. A. (2016). Eco and Green cities as new approaches for planning and developing cities in Egypt. Alexandria Engineering Journal, 55(1), 495-503. https://doi.org/10.1016/j.aej.2015.12.018

El-Chichakli, B., von Braun, J., Lang, C., Barben, D., \& Philp, J. (2016). Policy: Five cornerstones of a global bioeconomy. Nature News, 535(7611), 221. https://doi. org/10.1038/535221a

Haberl, H., \& Geissler, S. (2000). Cascade utilization of biomass: Strategies for a more efficient use of a scarce resource. Ecological Engineering, 16, 111-121. https://doi.org/10.1016/S0925-8574(00)00059-8

Hadad, S. (2019). Developing rural tourism in the context of sustainable bioeconomy - a Romanian perspective. Proceedings of the International Conference on Business Excellence, 13(1), 537-547. https://doi.org/10.2478/ picbe-2019-0047

Hernández, R., \& Céspedes, J. (2020). Bioeconomía: Una estrategia de sostenibilidad en la cuarta revolución industrial. Revista de Investigación e Innovación Agropecuaria y de Recursos Naturales, 7(2), 126-133.

ICT. (2009). Ley Organica del Instituto Costarricense de Turismo. https://www.ict. go.cr/es/documentos-institucionales/legislaci\%C3\%B3n-de-empresas/leyesy-reglamentos/610-ley-organica-del-instituto-costarricense-de-turismo/file. html

ICT. (2020). Divisas por concepto de Turismo (p. 5). Instituto Costarricesnse de Turismo. https://www.ict.go.cr/es/documentos-institucionales/ estad\%C3\%ADsticas/cifras-econ\%C3\%B3micas/costa-rica/960-divisas-porconcepto-de-turismo/file.html

Imbert, E., Ladu, L., Morone, P., \& Quitzow, R. (2017). Comparing policy strategies 
for a transition to a bioeconomy in Europe: The case of Italy and Germany. Energy Research \& Social Science, 33, 70-81. https://doi.org/10.1016/j. erss.2017.08.006

Kircher, M., Bott, M., \& Marienhagen, J. (2020). The Importance of Biotechnology for the Bioeconomy. En J. Pietzsch (Ed.), Bioeconomy for Beginners (pp. 105-128). Springer. https://doi.org/10.1007/978-3-662-60390-1_5

Lago, C., Herrera, I., Caldés, N., \& Lechón, Y. (2019). Chapter One-Nexus BioenergyBioeconomy. En C. Lago, N. Caldés, \& Y. Lechón (Eds.), The Role of Bioenergy in the Bioeconomy (pp. 3-24). Academic Press. https://doi.org/10.1016/B978-012-813056-8.00001-7

Lewandowski, I. (Ed.). (2018). Bioeconomy: Shaping the Transition to a Sustainable, Biobased Economy. Springer International Publishing. //www.springer.com/ gp/book/9783319681511

Lokko, Y., Heijde, M., Schebesta, K., Scholtès, P., Van Montagu, M., \& Giacca, M. (2018). Biotechnology and the bioeconomy-Towards inclusive and sustainable industrial development. New Biotechnology, 40, 5-10. https://doi. org/10.1016/j.nbt.2017.06.005

Maciejczak, M., \& Hofreiter, K. (2015). How To Define Bioeconomy? http://www. maciejczak.pl/download/15-4-Maciejczak.pdf

Martín, P. A. (2013). La situación del empleo en turismo rural en España. Estudios de economía aplicada, 31(1), 15.

MICITT. (2019). MICITT presentó X informe de Indicadores Nacionales de Ciencia, Tecnología e Innovación: Costa Rica 2018 | MICITT. https://www.micit. go.cr/noticias/micitt-presento-x-informe-indicadores-nacionales-cienciatecnologia-e-innovacion-costa-rica

MICITT. (2020). ¿Qué es un proyecto o iniciativa bioeconómica? Bionegocios. https://bionegocios.cr/que-es-un-proyecto-o-iniciativa-bioeconomica

OMPI. (2020, septiembre 2). Índice Mundial de Innovación 2020: Impacto previsto de la pandemia de COVID-19 en la innovación mundial; clasificación anual encabezada por Suiza, Suecia, los Estados Unidos, el Reino Unido y los Países Bajos. https://www.wipo.int/pressroom/es/articles/2020/article_0017.html 
Pyka, A. (2017). Dedicated innovation systems to support the transformation towards sustainability: Creating income opportunities and employment in the knowledge-based digital bioeconomy. Journal of Open Innovation: Technology, Market, and Complexity, 3(4), 27. https://doi.org/10.1186/s40852017-0079-7

RECOPE. (2018). Plan de descarbonización del sector transporte terrestre (p. 92) [Viabilidad]. Refinadora Costarricense de Petróleo S.A. https://www.recope. go.cr/wp-content/uploads/2019/03/RECOPE-PLAN-DE-DESCARBONIZACIONDEL-SECTOR-TRANSPORTE-TERRESTRE_VF.pdf

Rivera-Tenorio, M., \& Moya, R. (2020). Potential for pellet manufacturing with wood waste from construction in Costa Rica. Waste Management \& Research, 38(8), 886-895. https://doi.org/10.1177/0734242X19893022

Rodríguez, A. G. (2018). La bioeconomía y la nueva revolución industrial. 43.

Rodríguez, A. G., Mondaini, A. O., \& Hitschfeld, M. A. (2017). Bioeconomía en América Latina y el Caribe: Contexto global y regional y perspectivas. https:// repositorio.cepal.org//handle/11362/42427

Sanz-Hernández, A., Esteban, E., \& Garrido, P. (2019). Transition to a bioeconomy: Perspectives from social sciences. Journal of Cleaner Production, 224, 107-119. https://doi.org/10.1016/j.jclepro.2019.03.168

Scarlat, N., Dallemand, J.-F., Monforti-Ferrario, F., \& Nita, V. (2015). The role of biomass and bioenergy in a future bioeconomy: Policies and facts. Environmental Development, 15, 3-34. https://doi.org/10.1016/j. envdev.2015.03.006

Schütte, G. (2018). What kind of innovation policy does the bioeconomy need? New Biotechnology, 40, 82-86. https://doi.org/10.1016/j.nbt.2017.04.003

SCIJ. (2020). Sistema Costarricense de Información Jurídica. http://www.pgrweb. go.cr/scij/avanzada_pgr.aspx

Senara. (s. f.). 6877 Ley de creacion del SENARA. http://www.senara.or.cr/acerca_ del_senara/marco_legal/leyes_senara/6877\%20Ley\%20de\%20creacion\%20 del\%2OSENARA.pdf

Sistema Costarricense de Información Jurídica. (2009). Trámite de las 
Solicitudes de Registro de Agroquímicos No 8702. http://www. pgrweb.go.cr/scij/Busqueda/Normativa/Normas/nrm_texto_completo. aram1=NRTC\&nValor1=1\&nValor2=64772\&nValor3=75297\&strTipM=TC

Staffas, L., Gustavsson, M., McCormick, K., Staffas, L., Gustavsson, M., \& McCormick, K. (2013). Strategies and Policies for the Bioeconomy and Bio-Based Economy: An Analysis of Official National Approaches. Sustainability, 5(6), 2751-2769. https://doi.org/10.3390/su5062751

Steinberg, P. F., Steinberg, P. F., Steinberg, M. L. C. of S. and S. and P. of P. S. and E. P. P. F., Kraft, M. E., \& Kamieniecki, S. (2001). Environmental Leadership in Developing Countries: Transnational Relations and Biodiversity Policy in Costa Rica and Bolivia. MIT Press.

Turnheim, B., Berkhout, F., Geels, A., Hof, A., McMeekin, B., Nykvist, \& D.van Vuuren. (2015). Evaluating sustainability transitions pathways: Bridging analytical approaches to address governance challenges. https://www.sei. org/publications/evaluating-sustainability-transitions-pathways-bridginganalytical-approaches-to-address-governance-challenges/

UNED. (2012). Normativa_-_curso_Administración_Tributaria_Municipal.pdf. https://www.uned.ac.cr/extension/images/ifcmdl/Normativa_-_curso_ Administraci\%C3\%B3n_Tributaria_Municipal.pdf

UNESCO. (2020). How much does your country invest in R\&D? http://www.uis. unesco.org/_LAYOUTS/UNESCO/research-and-development-spending/indexen.html

Urmetzer, S., \& Pyka, A. (2017). Varieties of Knowledge-Based Bioeconomies. En S. Dabbert, I. Lewandowski, J. Weiss, \& A. Pyka (Eds.), Knowledge-Driven Developments in the Bioeconomy: Technological and Economic Perspectives (pp. 57-82). Springer International Publishing. https://doi.org/10.1007/978-3319-58374-7_4

Valli, M., Russo, H. M., Bolzani, V. S., Valli, M., Russo, H. M., \& Bolzani, V. S. (2018). The potential contribution of the natural products from Brazilian biodiversity to bioeconomy. Anais Da Academia Brasileira de Ciências, 90(1), 763-778. https:// doi.org/10.1590/0001-3765201820170653

Vogelpohl, T., \& Töller, A. E. (2021). Perspectives on the bioeconomy as an emerging policy field. Journal of Environmental Policy \& Planning, 23(2), 143-151. https:// 
doi.org/10.1080/1523908X.2021.1901394

Wield, D. (2013). Bioeconomy and the global economy: Industrial policies and bioinnovation. Technology Analysis \& Strategic Management, 25(10), 1209-1221. https://doi.org/10.1080/09537325.2013.843664 